\title{
TMD program at Jefferson Lab
}

\section{Tianbo Liu*}

Thomas Jefferson National Accelerator Facility, Newport News, VA 23606, USA

Department of Physics, Duke University, Durham, NC 27708, USA

E-mail: liutb@jlab.org

\begin{abstract}
Unveiling partonic structures of the nucleon is one of the missions of Jefferson Lab (JLab). Extracting the transverse momentum dependent parton distributions (TMDs), which provide threedimensional imagings of the nucleon in the momentum space, is a main physics program at JLab. Many explorations have been made in $6 \mathrm{GeV}$ experiments. A comprehensive TMD program with semi-inclusive deep inelastic scattering experiments in multiple halls will take place in the 12 $\mathrm{GeV}$ era, aiming at a profound understanding of the reaction mechanism and precise extractions of TMDs particularly in the valence quark region. Taking SoLID as an example, we show the impact of these experiments on TMD physics. Besides, some measurements can also advance the search for new physics beyond the standard model.
\end{abstract}

The 9th International workshop on Chiral Dynamics

17-21 September 2018

Durham, NC, USA

${ }^{*}$ Speaker. 


\section{Introduction}

The nucleon spin structure is one of the most active frontiers in nuclear and particle physics. In late 1980s, the EMC collaboration first measured the quark spin contribution to the proton spin and found that it only contributed a small fraction [1,2]. This discovery severely deviates from the naive quark model in which the proton spin is all from the quark spin. It is known as the "proton spin puzzle". During the last 30 years, many efforts have been made to understand the "missing spin" both theoretically and experimentally. Nowadays, the remaining proton spin is usually attributed to the gluon spin and orbital angular momenta, though there are still different decomposition appoaches: the canonical and the kinetic ones [3]. Experimentally, the quark spin part has been measured to a relatively good precision. The gluon spin part is started being known, particularly from STAR and PHENIX polarized proton-proton data. A first lattice QCD calculation of the gluon spin was also recently reported [4]. However, the orbital terms are still very poorly extracted. To fully understand the nucleon spin structure, one has to go beyond one dimension to access three dimensional imaging of the nucleon.

A unified framework of partonic structures of the nucleon is provided by the generalized transverse momentum dependent parton distributions (GTMDs) or via a Fourier transform by the Wigner distributions, which contain the most complete one parton distribution information. When the transverse coordinates or the transverse momenta are integrated out, they will respectively reduce to the transverse momentum dependent parton distributions (TMDs) and the generalized parton distributions (GPDs). If transverse variables are all integrated out, they will further reduce to the collinear parton distributions functions (PDFs). In this talk, we focus on the TMDs, which is one of the main physics programs at JLab during the $12 \mathrm{GeV}$ era.

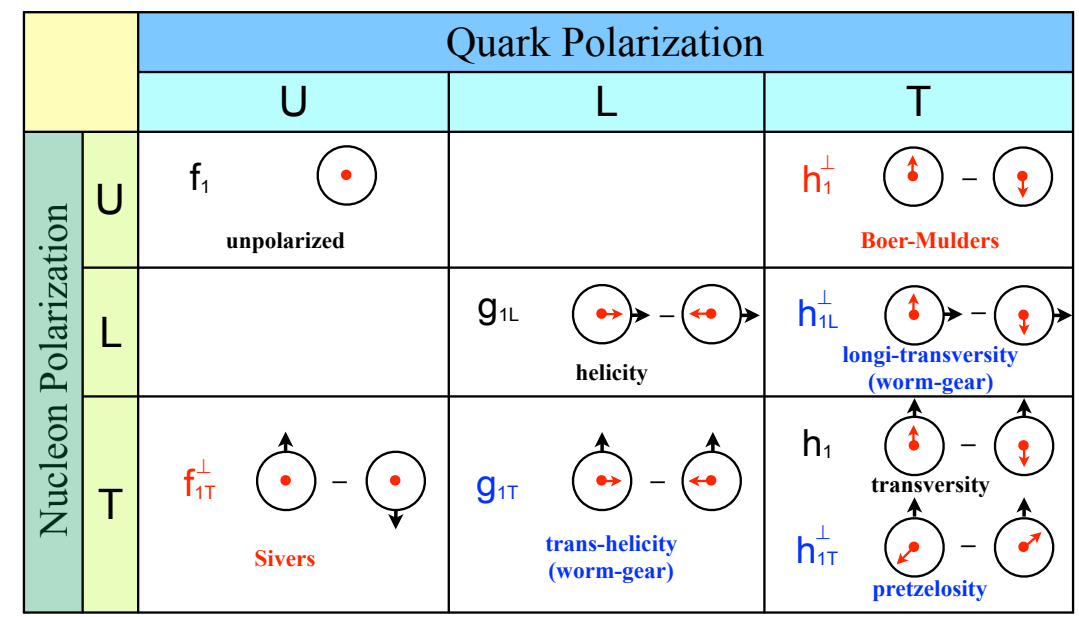

Figure 1: Leading twist quark TMDs of the nucleon. The black arrows represent the nucleon spin, and the red arrows represent the quark spin. U, L and T stand for unpolarized, longitudinal polarized and transversely polarized respectively. 


\section{SIDIS experiments at JLab}

The TMDs can be measured in various hard scattering processes, including the semi-inclusive deep inelastic scattering (SIDIS) and the Drell-Yan processes. Here we only focus on the SIDIS process. With one photon exchange approximation, its differential cross section can be expressed in terms of 18 structures functions according to different beam and target polarization configurations and azimuthal modulations of the final detected hadron [5]. Within the TMD factorization, the structure functions can be expressed as convolutions between TMD distribution functions and fragmentation functions. At the leading twist, eight quark TMDs are defined, as shown in Fig. 1. At the forward limit, only the unpolarized distribution, helicity distribution, and the transversity distribution survive. The red ones in Fig. 1 are known as the naively time-reversal odd TMDs, which arise from nontrivial Wilson lines that gaurantee the QCD gauge invariance [6], or physically from the final or initial state interactions [7, 8]. The QCD factorization theorem predicts a sign change of these time-reversal odd quark TMDs between SIDIS and Drell-Yan processes [9].

In the $6 \mathrm{GeV}$ era, some explorations have been made, such as the transversity experiment (E06010) in Hall A, which is the first polarized neutron SIDIS experiment [10]. The BigBite detector served as the electron arm, and the HRS detector served as the hadron arm. A $40 \mathrm{~cm}$ transversely polarized ${ }^{3} \mathrm{He}$ target is used as the effective polarized neutron target since its polarization is mainly from the neutron spin. Averaged in-beam polarization reached 55\%. Many results have been published from this experiment.

Nowadays, we are moving from explorations to precise measurements of TMDs. The $12 \mathrm{GeV}$ energy upgrade of CEBAF provides a unique opportunity to study quark TMDs, particularly in the valence quark region. A comprehensive TMD program using SIDIS measurements is planned at JLab-12. It takes advantages of the complementary capabilities of different detectors in Hall A, Hall B, and Hall C.

The SBS SIDIS experiment in Hall A will use the existing BigBite spectrometer and a new super BigBite spectrometer on a transversely polarized ${ }^{3} \mathrm{He}$ target. The super BigBite spectrometer with moderate solid angle and wide momentum acceptance takes place of the HRS used in the $6 \mathrm{GeV}$ experiment. The designed luminosity is increased roughly by a factor of five. Compared to the $6 \mathrm{GeV}$ experiment, the statistical figure-of-merit is roughly improved by a factor of one thousand.

The CLAS12 in Hall B is designed similar to the CLAS detector from $6 \mathrm{GeV}$. It is a general purpose detector for charged and neutral particles with large acceptance. Compared to CLAS, the designed luminosity is increased by one order of magnitude. The approved SIDIS experiments include unpolarized targets and longitudinally polarized targets as $\mathrm{NH}_{3}$ and $\mathrm{ND}_{3}$, and also a transversely polarized $\mathrm{HD}$-ice target.

Hall $\mathrm{C}$ in the $12 \mathrm{GeV}$ era hosts the existing high momentum spectrometer (HMS) and a new Super HMS (SHMS) detector, which is capable to reach more forward scattering angles with maximal momentum of $11 \mathrm{GeV}$. These small acceptance focusing magnetic spectrometers have well defined acceptance and excellent momentum and angular resolutions. The unpolarized SIDIS experiments in Hall $\mathrm{C}$ will measure cross sections with high precision at targeted kinematics to provide detailed understanding of the reaction mechanism, like the factorization framework and the L-T separations. They also provide a powerful cross check for data from large acceptance experiments. 
SoLID is proposed for Hall A beyond the $12 \mathrm{GeV}$ upgrade with broad physics interest. It is capable to handle high luminosities, about $10^{37} \mathrm{~cm}^{-2} \cdot \mathrm{s}^{-1}$ for the SIDIS setup, and combines large acceptance with a full azimuthal angle coverage, which can help a lot in the separation of different modulation terms in the SIDIS reaction. Three SIDIS experiments, respectively using transversely polarized $\mathrm{NH}_{3}$ target, longitudinally polarized ${ }^{3} \mathrm{He}$ target, and transversely polarized ${ }^{3} \mathrm{He}$ target, as well as some run-group experiments have been approved with high ratings, aiming at unprecedented precise measurements of quark TMDs in the valence region. Both $11 \mathrm{GeV}$ and $8.8 \mathrm{GeV}$ beams will be used for a wide kinematics coverage. The combination of data from proton and effective neutron targets can provide the flavor separation. The high statistics allows a four dimensional binning with high precision.

\section{The impact of SoLID SIDIS experiment}

To see the impact of the SIDIS program at JLab-12, we study the SoLID case for the expected improvement of TMD extractions. We take the transversity distribution as an example. The transversity distribution, which has both collinear and TMD definitions, is a leading twist distribution function that decribes the density of transversely polarized quarks in a transversely polarized nucleon. Unlike the helicity distribution, which describes the density of longitudinally polarized quarks in a longitudinally polarized nucleon, the transversity distribution is a chiral odd quantity. Hence it does not mix with gluons and is valence quark dominant.

As a chiral odd function the transversity distribution decouples from the inclusive DIS at the leading twist, which makes the measurement more difficult. One can instead extract the it from observables that include its convolution with another chiral odd quantity, like the Collins fragmentation function, dihadron fragmentation function, or another chiral odd distribution function. In SIDIS, one can measure the transversity distribution via a target transverse single spin asymmetry, the Collins asymmetry [6], which arises from its convolution with the Collins fragmentation functions within the TMD factorization.

We simulate the Collins asymmetry for SoLID SIDIS experiments using the parametrization in Ref. [11], which is a global analysis of Collin asymmetries in back-to-back dihadron production in $e^{+} e^{-}$annilations measured by Belle and BaBar and SIDIS data from HERMES, COMPASS and JLab Hall A including the TMD evolution effect. As shown in Fig. 2, SoLID SIDIS experiments are expected to reduce the uncertainties of up and down quark transversity distributions by about one order of magnitude [12]. In this study, both statistical and systematic uncertainties of world data and SoLID projected data are included and added in quadrature. The uncertainties of transversity distributions are estimated via the hessian matrix, which encodes the errors of each parameter and the error correlations of each pair of parameters.

In the parton model, one can evaluate the tensor charge from the transversity distribution via an integral,

$$
g_{T}^{q}=\int_{0}^{1}\left[h_{1}^{q}(x)-h_{1}^{\bar{q}}(x)\right] d x .
$$



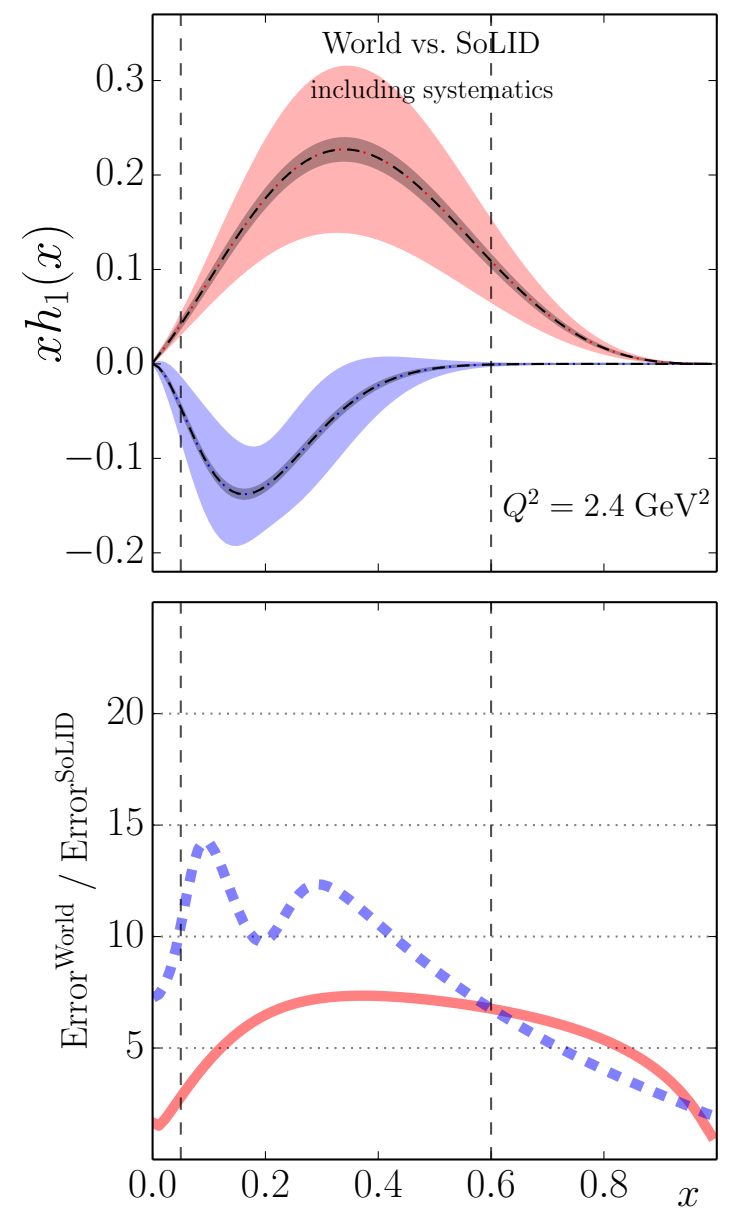

Figure 2: The impact of SoLID SIDIS experiments on the extraction of transversity distributions. The red stands for the up quark results, and the blue stands for the down quark results. The outer bands are uncertainties by fitting the world data. The inner bands are expected uncertainties after SoLID. The vertical dashed lines indicates the typical coverage of the SoLID data.

The tensor charge is a fundamental QCD quantity defined by the matrix element of the tensor current,

$$
\left\langle p, \sigma\left|\bar{\psi}_{q} i \sigma^{\mu v} \psi_{q}\right| p, \sigma\right\rangle=g_{T}^{q} \bar{u}(p, \sigma) i \sigma^{\mu v} u(p, \sigma) .
$$

It has been calculated in many phenomenological models [13, 14, 15, 16, 17, 18, 19, 20, 21, 22], and with some nonperturbative methods such as the Dyson-Schwinger equation [23, 24] and the Euclidean lattice simulation [25, 26, 27, 28, 29, 30]. It is also referred to as a benchmark of lattice QCD. As shown in Fig. 3, the SoLID SIDIS experiments will improve the precision of tensor charge extractions by about one order of magnitude, reaching the precision of lattice QCD calculations [12].

The tensor charge is an important quantity not only for nucleon structures but also for new physics. One example is the electric dipole moment (EDM). A permanent EDM of any particle with a nondegenerate ground state violates both parity $(\mathrm{P})$ and time-reversal $(\mathrm{T})$ symmetries. As- 


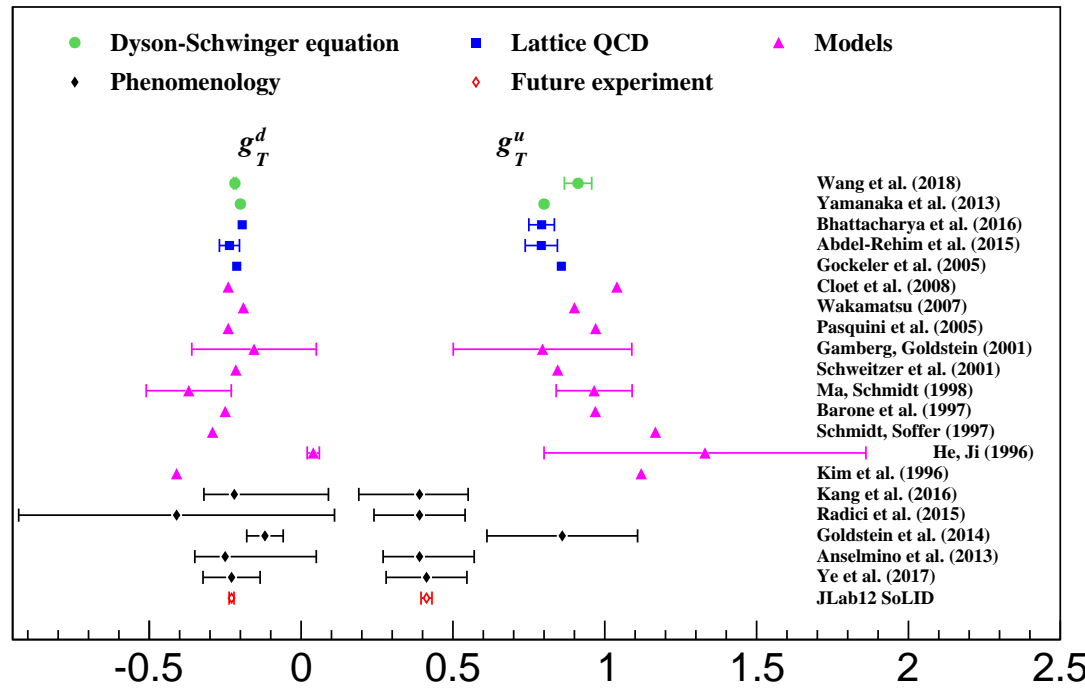

Figure 3: Tensor charge results. The round (green) points are from Dyson-Schwinger equation calculations [23, 24], the square (blue) points are from lattice QCD calculations [25, 26, 27, 28, 29, 30], the triangle (magenta) points are from model calculations [13, 14, 15, 16, 17, 18, 19, 20, 21, 22], the filled diamond (black) points are phenomenological extractions from data [11, 31, 32, 33, 12], and the hollow diamond (red) points are the projection of SoLID experiments based on the global analysis [12].
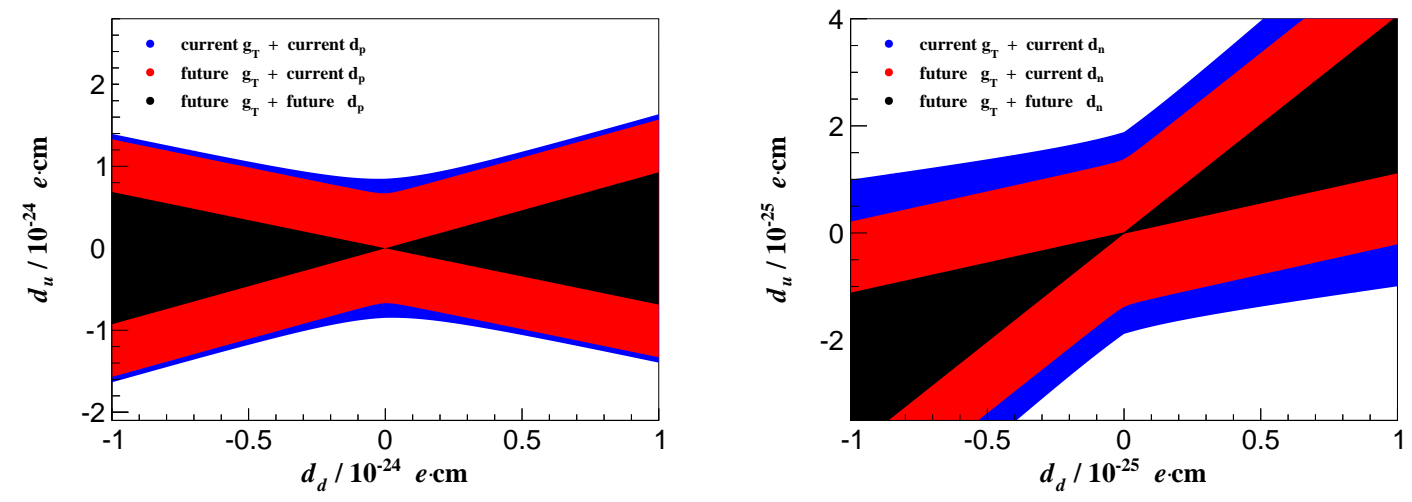

Figure 4: Constraints on quark EDMs from the proton EDM (left) and the neutron EDM (right) upper limits. The blue bands represent the current constraints, the red ones represents the constraints after SoLID, and the black ones represents the constraints after SoLID and next generation neutron and proton EDM experiments. 
suming CPT invariance, it is a signal of $\mathrm{CP}$ violation. Since the EDM of light quarks from the complex phase in the Cabibbo-Kobayashi-Maskawa (CKM) matrix is highly suppressed by the flavor changing interactions at the three-loop level, the Kobayashi-Maskawa mechanism only results in an extremely small quark EDM [34, 35, 36]. Thus, it is one of the most sensitive probes to new physics beyond the standard model. Due to the confinement property of strong interaction, however, the quark EDM is not directly measurable, but one can instead derive it from nucleon EDM measurements. The tensor charge plays as a bridge that relates the quark EDM to the nucleon EDM.

We study the experimental constraint on quark EDMs by combining the nucleon EDM measurements and the tensor charge extractions. The current upper limit on the neutron EDM from direct measurements is $3.0 \times 10^{-26} e \cdot \mathrm{cm}(90 \%$ C.L.) [37]. The current upper limit on the proton EDM is derived from the mercury atomic EDM limit with the Schiff moment method [38], and the most recent measurement sets the upper limit of ${ }^{199} \mathrm{Hg}$ atoms to $7.4 \times 10^{-30} e \cdot \mathrm{cm}(95 \%$ C.L.) [39]. Including the uncertainty from the nuclear effect, we get the derived upper limit on the proton EDM as $2.6 \times 10^{-25} e \cdot \mathrm{cm}[40]$. With the tensor charge extracted from the global analysis of the Collins asymmetry, one can derive the constraints on quark EDMs from the proton and neutron EDM measurements. As the strange quark transversity is set to zero in the global fit, we adopt the value from a lattice QCD calculation [41] to include its effect. The derived constraints on quark EDMs are shown in Fig. 4. To improve the constraint, efforts from both tensor charge extractions and from nucleon EDM measurements are required. As mentioned above, SoLID SIDIS experiments will significantly reduce the tensor charge uncertainty. On the other hand, the next generation neutron EDM experiments will improve the sensitivity by two orders of magnitude to $10^{-28} e \cdot \mathrm{cm}$ [42], and the proposed storage ring proton EDM experiment will have direct measurement of the proton EDM with the sensitivity of $10^{-29} e \cdot \mathrm{cm}$ [43]. The impact of these future experiments are shown in Fig. 4.

Combining the results from the proton EDM and neutron EDM limits, we can have the flavor separation. The current upper limits are $1.27 \times 10^{-24} e \cdot \mathrm{cm}$ for the up quark and $1.17 \times 10^{-24} e$. $\mathrm{cm}$ for the down quark, where $10 \%$ isospin symmetry breaking uncertainties are included. After SoLID SIDIS experiments and the next generation nucleon EDM measurements, the upper limits are expected to be improved by about three orders of magnitude [40].

Apart from the study of the transversity distribution with the Collins asymmetry, we take another example, the Sivers asymmetry, which arises from the convolution between the Sivers function and unpolarized fragmentation function. Similar to the transversity case, a global fit to world data is used to simulate SoLID Sivers asymmetry values. In this study, we adopt the Monte Carlo analysis using the nested sampling algorithm [44]. The preliminary results are shown in Fig. 5.

\section{Summary}

The lepton scattering is a powerful tool to probe nucleon internal structures. Three dimensional imagings of the nucleon can help understand the remaining puzzle to the proton spin and uncover rich dynamics of the strong interaction. Many efforts have been made in JLab $6 \mathrm{GeV}$ SIDIS experiments. A rich TMD program with SIDIS experiments in multiple halls will happen 

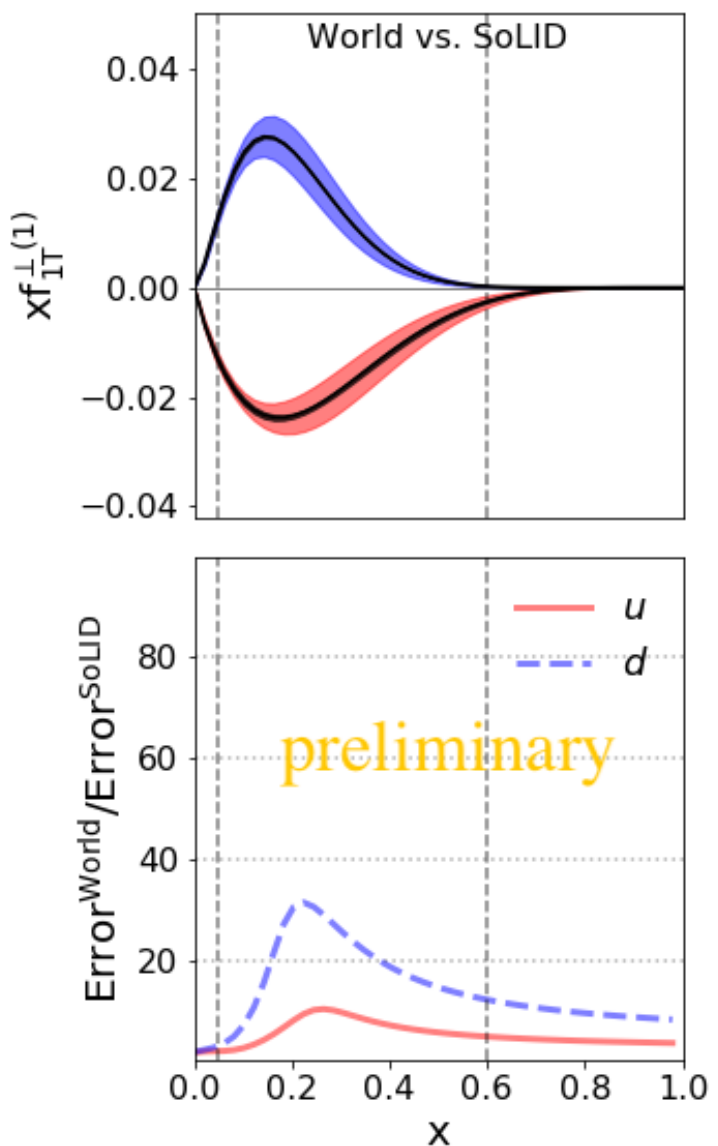

Figure 5: The impact of SoLID SIDIS experiments on the extraction of the Sivers function. The inner bands are expected uncertainties after SoLID. The vertical dashed lines indicates the typical coverage of the SoLID data.

in the $12 \mathrm{GeV}$ era. It will have significant improvement of TMD extractions in the valence quark region. Some measurements, such as the tensor charge from the transversity distribution, can also help the search for new physics beyond the standard model.

\section{Acknowledgments}

This work is supported in part by U.S. Department of Energy under contracts No. DE-FG0203ER41231 and No. DE-AC05-06OR23177.

\section{References}

[1] J. Ashman et al. (European Muon Collaboration), Phys. Lett. B 206, 364 (1988).

[2] J. Ashman et al. (European Muon Collaboration), Nucl. Phys. B 328, 1 (1989).

[3] E. Leader and C. Lorcé, Phys. Rept. 541, no. 3, 163 (2014). 
[4] Y.-B. Yang, R. S. Sufian, A. Alexandru, T. Draper, M. J. Glatzmaier, K.-F. Liu and Y. Zhao, Phys. Rev. Lett. 118, no. 10, 102001 (2017).

[5] A. Bacchetta, M. Diehl, K. Goeke, A. Metz, P. J. Mulders and M. Schlegel, J. High Energy Phys. 02 (2007) 093.

[6] J. C. Collins, Phys. Lett. B 536, 43 (2002).

[7] S. J. Brodsky, D. S. Hwang and I. Schmidt, Nucl. Phys. B 642, 344 (2002).

[8] S. J. Brodsky, D. S. Hwang and I. Schmidt, Phys. Lett. B 530, 99 (2002).

[9] J. C. Collins and A. Metz, Phys. Rev. Lett. 93, 252001 (2004).

[10] X. Qian et al. (Jefferson Lab Hall A Collaboration), Phys. Rev. Lett. 107, 072003 (2011).

[11] Z. B. Kang, A. Prokudin, P. Sun and F. Yuan, Phys. Rev. D 93, no. 1, 014009 (2016).

[12] Z. Ye et al., Phys. Lett. B 767, 91 (2017).

[13] I. C. Cloet, W. Bentz and A. W. Thomas, Phys. Lett. B 659, 214 (2008).

[14] M. Wakamatsu, Phys. Lett. B 653, 398 (2007).

[15] B. Pasquini, M. Pincetti and S. Boffi, Phys. Rev. D 72, 094029 (2005).

[16] L. P. Gamberg and G. R. Goldstein, Phys. Rev. Lett. 87, 242001 (2001).

[17] P. Schweitzer, D. Urbano, M. V. Polyakov, C. Weiss, P. V. Pobylitsa and K. Goeke, Phys. Rev. D 64, 034013 (2001).

[18] B.-Q. Ma and I. Schmidt, J. Phys. G 24, L71 (1998).

[19] V. Barone, T. Calarco and A. Drago, Phys. Lett. B 390, 287 (1997).

[20] I. Schmidt and J. Soffer, Phys. Lett. B 407, 331 (1997).

[21] H.-x. He and X. Ji, Phys. Rev. D 54, 6897 (1996).

[22] H. C. Kim, M. V. Polyakov and K. Goeke, Phys. Lett. B 387, 577 (1996).

[23] Q. W. Wang, S. X. Qin, C. D. Roberts and S. M. Schmidt, Phys. Rev. D 98, no. 5, 054019 (2018).

[24] N. Yamanaka, T. M. Doi, S. Imai and H. Suganuma, Phys. Rev. D 88, 074036 (2013).

[25] T. Bhattacharya, V. Cirigliano, S. Cohen, R. Gupta, H. W. Lin and B. Yoon, Phys. Rev. D 94, no. 5, 054508 (2016).

[26] A. Abdel-Rehim et al., Phys. Rev. D 92, 114513 (2015) [Erratum: D 93, 039904 (2016)].

[27] M. Gockeler et al. (QCDSF and UKQCD Collaborations), Phys. Lett. B 627, 113 (2005).

[28] G. S. Bali et al., Phys. Rev. D 91, no. 5, 054501 (2015).

[29] J. R. Green, J. W. Negele, A. V. Pochinsky, S. N. Syritsyn, M. Engelhardt and S. Krieg, Phys. Rev. D 86, 114509 (2012).

[30] Y. Aoki, T. Blum, H. W. Lin, S. Ohta, S. Sasaki, R. Tweedie, J. Zanotti and T. Yamazaki, Phys. Rev. D 82, 014501 (2010).

[31] M. Radici, A. Courtoy, A. Bacchetta and M. Guagnelli, J. High Energy Phys. 05 (2015) 123.

[32] G. R. Goldstein, J. O. Gonzalez Hernandez and S. Liuti, arXiv:1401.0438 [hep-ph]. 
[33] M. Anselmino, M. Boglione, U. D’Alesio, S. Melis, F. Murgia and A. Prokudin, Phys. Rev. D 87, 094019 (2013).

[34] A. Czarnecki and B. Krause, Phys. Rev. Lett. 78, 4339 (1997).

[35] M. B. Gavela, A. Le Yaouanc, L. Oliver, O. Pene, J. C. Raynal and T. N. Pham, Phys. Lett. 109B, 215 (1982).

[36] I. B. Khriplovich and A. R. Zhitnitsky, Phys. Lett. 109B, 490 (1982).

[37] J. M. Pendlebury et al., Phys. Rev. D 92, no. 9, 092003 (2015).

[38] V. F. Dmitriev and R. A. Sen'kov, Phys. Rev. Lett. 91, 212303 (2003).

[39] B. Graner, Y. Chen, E. G. Lindahl and B. R. Heckel, Phys. Rev. Lett. 116, 161601 (2016) [Erratum: 119, 119901 (2017)].

[40] T. Liu, Z. Zhao and H. Gao, Phys. Rev. D 97, no. 7, 074018 (2018).

[41] T. Bhattacharya, V. Cirigliano, R. Gupta, H. W. Lin and B. Yoon, Phys. Rev. Lett. 115, no. 21, 212002 (2015).

[42] Conceptual Design Report for the nEDM Project, https://www.phy.ornl.gov/groups/neutrons/nEDM_CDR.pdf.

[43] V. Anastassopoulos et al., Rev. Sci. Instrum. 87, no. 11, 115116 (2016); Storage Ring EDM Collaboration, A proposal to measure the proton electric dipole moment with $10^{-29} \mathrm{e} \cdot \mathrm{cm}$ sensitivity, http://www.bnl.gov/edm/.

[44] F. Feroz, M. P. Hobson, E. Cameron and A. N. Pettitt, arXiv:1306.2144 [astro-ph. IM]. 